\title{
Combinatorial identities in the context of hypercomplex function theory
}

\author{
I. Cação ${ }^{1}$, M. I. Falcão ${ }^{2}$ and H. R. Malonek ${ }^{1, a)}$ \\ ${ }^{1}$ CIDMA and Department of Mathematics, University of Aveiro, Campus Universitário de Santiago, 3810-193 \\ Aveiro, Portugal \\ ${ }^{2}$ Centre of Mathematics and Department of Mathematics and Applications, University of Minho, Campus de Gualtar, \\ 4710-057 Braga, Portugal
}

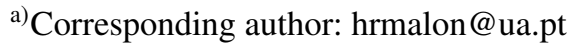

\begin{abstract}
Recently, the authors have shown that a certain combinatorial identity in terms of generators of quaternions is related to a particular sequence of rational numbers (Vietoris' number sequence). This sequence appeared for the first time in a theorem by Vietoris (1958) and plays an important role in harmonic analysis and in the theory of stable holomorphic functions in the unit disc. We present a generalization of that combinatorial identity involving an arbitrary number of generators of a Clifford algebra. The result reveals new insights in combinatorial phenomena in the context of hypercomplex function theory.
\end{abstract}

\section{INTRODUCTION AND BASIC NOTATIONS}

The identity is verified ... by operations in which properties of the binomial coefficients are employed. Combinatorialists use recurrence, generating functions, and such transformations as the Vandermonde convolutions; others, to my horror, use contour integrals, differential equations, and other resources of mathematical analysis.

John Riordan

(in: Combinatorial Identities, Wiley, 1968)

Combinatorics as a branch of mathematics is concerned with the study of finite or countable discrete structures and has recently enjoyed a rapid growth, partially influenced by new connections to other fields like algebra, probability theory, topology, geometry or their applications. The powerful algebraic computational tools for manipulating combinatorial expressions, unknown in the time of J. Riordan's book, essentially contribute to the same development. Our aim is to show that not only the classical resources of complex analysis, horrifying J. Riordan, are very useful tools for detecting or verifying combinatorial identities, but even some facts about Clifford algebra-valued polynomials in several hypercomplex variables can play an important role in this context.

As usual, we consider an orthonormal basis $\left\{e_{1}, e_{2}, \cdots, e_{n}\right\}$ of the Euclidean vector space $\mathbb{R}^{n}$ with a noncommutative product according to the multiplication rules $e_{k} e_{l}+e_{l} e_{k}=-2 \delta_{k l}, k, l=1, \cdots, n$, where $\delta_{k l}$ is the Kronecker symbol. Then the set $\left\{e_{A}: A \subseteq\{1, \cdots, n\}\right\}$ with $e_{A}=e_{h_{1}} e_{h_{2}} \cdots e_{h_{r}}, 1 \leq h_{1}<\cdots<h_{r} \leq n, e_{\emptyset}=e_{0}=1$, forms a basis of the $2^{n}$-dimensional Clifford algebra $C \ell_{0, n}$ over $\mathbb{R}$. We embed $\mathbb{R}^{n+1}$ in $C \ell_{0, n}$ by identifying $\left(x_{0}, x_{1}, \cdots, x_{n}\right) \in$ $\mathbb{R}^{n+1}$ with $x=x_{0}+\underline{x} \in \mathcal{A}:=\operatorname{span}_{\mathbb{R}}\left\{1, e_{1}, \ldots, e_{n}\right\} \subset C \ell_{0, n}$. Here $x_{0}=\mathcal{S}(x)$ and $\underline{x}=\mathcal{V}(x)=e_{1} x_{1}+\cdots+e_{n} x_{n}$ are the so-called scalar resp. vector part of the paravector $x \in \mathcal{A}$. The conjugate of $x$ is given by $\bar{x}=x_{0}-\underline{x}$ and its norm by $|x|=(x \bar{x})^{\frac{1}{2}}=\left(x_{0}^{2}+x_{1}^{2}+\cdots+x_{n}^{2}\right)^{\frac{1}{2}}$. Often, $\left\{e_{1}, e_{2}, \cdots, e_{n}\right\}$ are called the imaginary units or generators of the Clifford algebra $C \ell_{0, n}$. Obviously, we can identify the case $n=1$ with the complex algebra case by $i:=e_{1}$ and the case $n=2$, where $e_{1}=\mathbf{i}, e_{2}=\mathbf{j}, e_{1} e_{2}=\mathbf{k}$, with the quaternion algebra case $C \ell_{0,2} \cong \mathbb{H}$. Notice that, in particular, we have $\left(e_{1} e_{2}\right)^{2}=\mathbf{k}^{2}=\mathbf{i j k}=-1$.

We need also a generalized Cauchy-Riemann operator in $\mathbb{R}^{n+1}, n \geq 1$, defined by $\bar{\partial}:=\frac{1}{2}\left(\partial_{0}+\partial_{\underline{x}}\right)$ and its conjugate $\partial:=\frac{1}{2}\left(\partial_{0}-\partial_{\underline{x}}\right)$ where $\partial_{0}:=\frac{\partial}{\partial x_{0}}$ and $\partial_{\underline{x}}:=e_{1} \frac{\partial}{\partial x_{1}}+\cdots+e_{n} \frac{\partial}{\partial x_{n}}$. $C^{1}$-functions $f$ in the kernel of $\bar{\partial}$, i.e. with $\bar{\partial} f=0$ (resp. $f \bar{\partial}=0$ ) are called left Clifford holomorphic (resp. right Clifford holomorphic), [1], or left resp. right monogenic [2]. 
We suppose that $f$ is hypercomplex-differentiable in $\Omega$ in the sense of [3], that is, it has a uniquely defined areolar derivative $f^{\prime}$ in each point of $\Omega$. Then, $f$ is real-differentiable and $f^{\prime}$ can be expressed by the conjugate generalized Cauchy-Riemann operator as $f^{\prime}=\partial f$. Since a hypercomplex differentiable function belongs also to the kernel of $\bar{\partial}$, one has $f^{\prime}=\partial_{0} f=-\partial_{\underline{x}} f$ like in the complex case.

In the following we intensively use the embedding of the non-commutative Clifford algebra product into an $n$ - nary symmetric product (see [4] and more detailed [5]):

Definition 1 (Symmetric Product) Let $V_{+}$, be a commutative or non-commutative ring, $a_{k} \in V, k=1, \ldots, n$, then the " $\times$ "-product is defined by

$$
a_{1} \times a_{2} \times \cdots \times a_{n}=\frac{1}{n !} \sum_{\pi\left(s_{1}, \ldots, s_{n}\right)} a_{s_{1}} a_{s_{2}} \cdots a_{s_{n}}
$$

where the sum runs over all permutations of all $\left(s_{1}, \ldots, s_{n}\right)$. Moreover, if the factor $a_{j}$ occurs $\mu_{j}$-times in $(1)$, we briefly write $a_{1} \times a_{2} \times \cdots \times a_{n}=a_{1}{ }^{\mu_{1}} \times a_{2}{ }^{\mu_{2}} \times \cdots \times a_{n}{ }^{\mu_{n}}$ and set parentheses if the powers are understood in the ordinary way.

Let us now recall the announced combinatorial identity which was proved in [6] and involves the generators of quaternions.

Theorem $1 \quad$ Let $\mathbf{i}$ and $\mathbf{j}$ be the generators of $\mathbb{H}$. Then the following combinatorial identity is true

$$
\left(\begin{array}{c}
k \\
\left\lfloor\frac{k}{2}\right\rfloor
\end{array}\right)\left[\sum_{s=0}^{k}\left(\begin{array}{l}
k \\
s
\end{array}\right)\left(\mathbf{i}^{k-s} \times \mathbf{j}^{s}\right)^{2}\right]=(-2)^{k}, \quad k \geq 0 .
$$

It follows immediately that

$$
\frac{1}{2^{k}}\left(\begin{array}{c}
k \\
\left\lfloor\frac{k}{2}\right\rfloor
\end{array}\right)=(-1)^{k}\left[\sum_{s=0}^{k}\left(\begin{array}{l}
k \\
s
\end{array}\right)\left(\mathbf{i}^{k-s} \times \mathbf{j}^{s}\right)^{2}\right]^{-1} .
$$

The combinatorial identity (2) was proved in [6] by calculating directly the values of the symmetric products $\mathbf{i}^{k-s} \times \mathbf{j}^{s}$. It is an easy task to recognize the left hand side of (3) as the sequence of rational numbers

$$
1, \frac{1}{2}, \frac{1}{2}, \frac{3}{8}, \frac{3}{8}, \frac{5}{16}, \frac{5}{16}, \frac{35}{128}, \frac{35}{128}, \frac{63}{256}, \frac{63}{256}, \frac{231}{1024}, \frac{231}{1024}, \ldots
$$

which appeared in the context of positive trigonometric sums in the celebrated paper of L. Vietoris [7]. Askey's version [8] of Vietoris' theorem is the following:

\section{Theorem 2 (L. Vietoris)}

$$
\sum_{k=1}^{n} a_{k} \sin k \theta>0, \quad 0<\theta<\pi, \text { and } \sum_{k=0}^{n} a_{k} \cos k \theta>0, \quad 0 \leq \theta<\pi,
$$

where

$$
a_{2 k}=a_{2 k+1}=\frac{\left(\frac{1}{2}\right)_{k}}{k !}, \quad k=0,1, \ldots,
$$

with $(\cdot)_{k}$ as the raising factorial in the classical form of the Pochhammer symbol.

The coefficients in the sine sum (starting with the index $k=1$ ) used in Askey's as well as in Vietoris' original version are exactly the elements of (4). In the next section we show that (2) can be generalized to a combinatorial identity involving the generators of an arbitrary Clifford algebra $C \ell_{0, n}$, but this time by applying properties of a sequence of hypercomplex Appell polynomials (see [10] and [11], where the concept of Appell sequences in hypercomplex context was introduced). 


\section{A COMBINATORIAL IDENTITY IN TERMS OF GENERATORS OF A CLIFFORD ALGEBRA}

We show now that our goal, i.e. the generalization of (2), can be achieved by combining several results previously obtained in other contexts and not as a direct generalization with the same methods as (2) was obtained in [6]. We start with recalling a theorem proved in [9].

Theorem 3 Let $n \geq 1$ be fixed and for each $k=0,1, \ldots$, consider the sets of real numbers $\left\{T_{s}^{k}(n)\right\}_{(0 \leq s \leq k)}$ defined by

$$
T_{s}^{k}(n):=\left(\begin{array}{l}
k \\
s
\end{array}\right) \frac{\left(\frac{n+1}{2}\right)_{k-s}\left(\frac{n-1}{2}\right)_{s}}{(n)_{k}} .
$$

After that, build for $|x|<1$, the homogeneous of degree $k$ polynomials in $x$ and $\bar{x}$

$$
\mathcal{P}_{k}^{n}(x):=\sum_{s=0}^{k} T_{s}^{k}(n) x^{k-s} \bar{x}^{s}
$$

Then the series

$$
\mathcal{P}_{0}^{n}(x)+\frac{n}{1 !} \mathcal{P}_{1}^{n}(x)+\frac{n(n+1)}{2 !} \mathcal{P}_{2}^{n}(x)+\frac{n(n+1)(n+2)}{3 !} \mathcal{P}_{3}^{n}(x)+\cdots
$$

is the hypercomplex generalized geometric series whose sum in $|x|<1$ is given by

$$
g(x)=(1-x)^{-1}|1-x|^{1-n}=\sum_{k=0}^{+\infty} \frac{(n)_{k}}{k !} \mathcal{P}_{k}^{n}(x) .
$$

From one side, since $\left.\mathcal{P}_{k}^{n}(x)\right|_{\underline{x}=0}=x_{0}^{k}$ it is evident, that $\mathcal{P}_{k}^{n}(1)=1, \quad k=0,1, \ldots$. From the other side, (6) implies for $x_{0}=0$ that

$$
\mathcal{P}_{k}^{n}(\underline{x})=\sum_{s=0}^{k}(-1)^{s} T_{s}^{k}(n) \underline{x}^{k}=c_{k}(n) \underline{x}^{k}
$$

where

$$
c_{k}(n):=\sum_{s=0}^{k}(-1)^{s} T_{s}^{k}(n)
$$

This formula appeared for the first time in $[10,11]$ for the particular case $n=2$, including the determination of $c_{k}(2)$ in the form

$$
c_{k}(2)=(-1)^{k}\left[\sum_{s=0}^{k}\left(\begin{array}{l}
k \\
s
\end{array}\right)\left(e_{1}^{k-s} \times e_{2}^{s}\right)^{2}\right]^{-1} .
$$

Formula (7) was obtained in $[10,11]$ by treating $\mathcal{P}_{k}^{2}(x)$ as a sequence of monogenic Appell polynomials in two hypercomplex variables according to the approach described in [12]. Its right side is nothing else than the right side of formula (3) in another notation. This observation indicates the way for the further reasoning. For the case of $n$ hypercomplex variables [12] allows to recognize the generalization of (7) in the form

$$
c_{k}(n)=(-1)^{k}\left[\sum_{|v|=k}\left(\begin{array}{l}
k \\
v
\end{array}\right)\left(e_{1}^{\nu_{1}} \times \cdots \times e_{n}^{\nu_{n}}\right)^{2}\right]^{-1},
$$

where $v=\left(v_{1}, \ldots v_{n}\right)$ is a multi-index and $\left(\begin{array}{l}k \\ v\end{array}\right)=\frac{k !}{v !}$. In $[10,11]$ one can also find the explicit values of $c_{k}(2)$

$$
c_{k}(2)=\left\{\begin{array}{l}
\frac{k ! !}{(k+1) ! !}, \text { if } k \text { is odd } \\
c_{k-1}(2), \text { if } k \text { is even }
\end{array}\right.
$$


which, in turn, can be written in the form

$$
c_{k}(2)=\frac{1}{2^{k}}\left(\begin{array}{c}
k \\
\left\lfloor\frac{k}{2}\right\rfloor
\end{array}\right)=\frac{\left(\frac{1}{2}\right)_{\left\lfloor\frac{k+1}{2}\right\rfloor}}{(1)_{\left\lfloor\frac{k+1}{2}\right\rfloor}}
$$

used in (3) in terms of the generalized central binomial coefficient resp. in terms of Pochhammer symbols as in (5). Obviously, (8) is the special $n=2$ case of

$$
c_{k}(n)=\left\{\begin{array}{l}
\frac{k ! !(n-2) ! !}{(n+k-1) ! !}, \text { if } k \text { is odd } \\
c_{k-1}(n), \text { if } k \text { is even }
\end{array}\right.
$$

These explicit values of $c_{k}(n)$ have been determined in [13, Th. 3.9]. Some cumbersome but elementary calculations show that an analogue suggested by formula (9) also exists for $c_{k}(n)$ in (10), namely

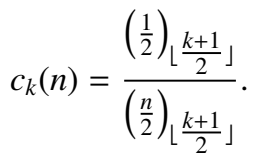

Combining all relevant formulas together we end up with the generalization of (2) in the form

$$
\left(\begin{array}{c}
k \\
\left\lfloor\frac{k}{2}\right\rfloor
\end{array}\right)\left[\sum_{|v|=k}\left(\begin{array}{l}
k \\
v
\end{array}\right)\left(e_{1}^{v_{1}} \times e_{2}^{v_{2}} \times \cdots \times e_{n}^{v_{n}}\right)^{2}\right]=(-2)^{k}\left(\begin{array}{c}
\left\lfloor\frac{k+1}{2}\right\rfloor+\frac{n-2}{2} \\
\left\lfloor\frac{k+1}{2}\right\rfloor
\end{array}\right), \quad k \geq 0 .
$$

\section{ACKNOWLEDGMENTS}

The work of the first and third authors was supported by Portuguese funds through the CIDMA - Center for Research and Development in Mathematics and Applications, and the Portuguese Foundation for Science and Technology ("FCT-Fundação para a Ciência e Tecnologia"), within project PEst-OE/MAT/UI4106/2013. The work of the second author was supported by Portuguese funds through the CMAT - Centre of Mathematics and FCT within the Project UID/MAT/00013/2013.

\section{REFERENCES}

[1] K. Gürlebeck, K. Habetha, and W. Sprößig, Holomorphic functions in the plane and n-dimensional space (Birkhäuser Verlag, Basel, 2008), pp. xiv+394, translated from the 2006 German original.

[2] F. Brackx, R. Delanghe, and F. Sommen, Clifford analysis (Pitman, Boston-London-Melbourne, 1982).

[3] K. Gürlebeck and H. Malonek, Complex Variables Theory Appl. 39, 199-228 (1999).

[4] H. Malonek, Complex Variables, Theory Appl. 15, 181-191 (1990).

[5] C. Cruz, M. I. Falcão, and H. R. Malonek, "About Pascal's tetrahedron with hypercomplex entries," in AIP Conference Proceedings, Vol. 1558 (2013), pp. 509-512.

[6] I. Cação, M. I. Falcão, and H. R. Malonek, "On Vietoris' number sequence and combinatorial identities with quaternions," in CMMSE 2017: Proceedings of the 17th International Conference on Computational and Mathematical Methods in Science and Engineering, edited by J. Vigo-Aguiar (Almería, Spain, 2017), pp. 480-488.

[7] L. Vietoris, Sitzungsber. Österr. Akad. Wiss 167, 125-135 (1958).

[8] R. Askey and J. Steinig, Transactions AMS 187, 295-307 (1974).

[9] I. Cação, M. I. Falcão, and H. Malonek, Complex Anal. Oper. Theory 11, 1059-1076 (2017).

[10] H. R. Malonek and M. I. Falcão, "Special monogenic polynomials - Properties and applications," in AIP Conference Proceedings, Vol. 936 (2007), pp. 764-767.

[11] M. I. Falcão and H. R. Malonek, "Generalized exponentials through Appell sets in $\mathbb{R}^{n+1}$ and Bessel functions," in AIP Conference Proceedings, Vol. 936 (2007), pp. 738-741.

[12] H. Malonek, in Clifford Algebras and Potential Theory, Vol. 7, edited by S.-L. Eriksson (University of Joensuu, 2004), pp. 111-150.

[13] M. I. Falcão and H. R. Malonek, Opuscula Mathematica 32, 661-673 (2012). 